\section{The International Hereditary Thrombotic Thrombocytopenic Purpura Registry: key findings at enrollment until 2017}

\author{
Hendrika A. van Dorland,,$^{1,2}$ Magnus Mansouri Taleghani, ${ }^{1}$ Kazuya Sakai, ${ }^{3}$ \\ Kenneth D. Friedman, ${ }^{4}$ James N. George,${ }^{5}$ Ingrid Hrachovinova, ${ }^{6}$ \\ Paul N. Knöbl, ${ }^{7}$ Anne Sophie von Krogh, ${ }^{8}$ Reinhard Schneppenheim, ${ }^{9}$ \\ Isabella Aebi-Huber, ${ }^{1,2}$ Lukas Bütikofer, ${ }^{10}$ Carlo R. Largiadèr, ${ }^{11}$ \\ Zuzana Cermakova, ${ }^{12}$ Koichi Kokame, ${ }^{13}$ Toshiyuki Miyata, ${ }^{13,14}$ Hideo Yagi,, 15 \\ Deirdra R. Terrell, ${ }^{5}$ Sara K. Vesely, ${ }^{5}$ Masanori Matsumoto, ${ }^{3}$ \\ Bernhard Lämmle, ${ }^{1,16}$ Yoshihiro Fujimura ${ }^{3,17}$ and Johanna A. Kremer Hovinga; ${ }^{1,2}$ \\ Hereditary TTP Registry
}

${ }^{1}$ Department of Hematology and Central Hematology Laboratory, Inselspital, Bern University Hospital, Bern, Switzerland; ${ }^{2}$ Department for BioMedical Research, University of Bern, Bern, Switzerland; ' ${ }^{2}$ Department of Blood Transfusion Medicine, Nara Medical University, Kashihara, Japan; ${ }^{4}$ Division of Hematology and Oncology, Medical College of Wisconsin, Milwaukee, WI, USA; ${ }^{5}$ Department of Biostatistics Epidemiology, University of Oklahoma Health Sciences Center, Oklahoma City, OK, USA; ${ }^{6}$ NRL for Hemostasis, Institute of Hematology and Blood Transfusion, Prague, Czech Republic; ${ }^{7}$ Division of Hematology and Hemostasis, Department of Medicine 1, Medical University of Vienna, Austria; ${ }^{8}$ Department of Hematology, St Olavs Hospital, Trondheim University Hospital, Trondheim, Norway; ${ }^{9}$ Department of Paediatric Haematology and Oncology, University Medical Centre Hamburg-Eppendorf, Hamburg, Germany; ${ }^{10} \mathrm{CTU}$ Bern, University of Bern, Bern, Switzerland; ${ }^{11}$ University Institute of Clinical Chemistry, Inselspital, Bern University Hospital, Bern, Switzerland; ${ }^{12}$ Blood Center, University Hospital Ostrava, Ostrava, Czech Republic; ${ }^{13}$ Department of Molecular Pathogenesis, National Cerebral and Cardiovascular Center, Suita, Japan; ${ }^{14}$ Department of Cerebrovascular Medicine, National Cerebral and Cardiovascular Center, Suita, Japan; ${ }^{15}$ Department of Hematology, Nara Prefecture General Medical Center, Nara, Japan; ${ }^{16}$ Center for Thrombosis and Hemostasis, University

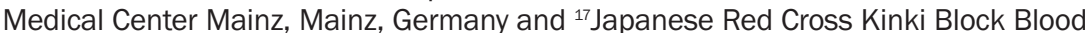
Center, Ibaraki, Osaka, Japan

\section{ABSTRACT}

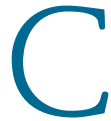

ongenital thrombotic thrombocytopenic purpura is an autosomal recessive inherited disease with a clinically heterogeneous course and an incompletely understood genotype-phenotype correlation. In 2006, the Hereditary TTP Registry started recruitment for a study which aimed to improve the understanding of this ultra-rare disease. The objective of this study is to present characteristics of the cohort until the end of 2017 and to explore the relationship between overt disease onset and ADAMTS13 activity with emphasis on the recurring ADAMTS13 c.4143_4144dupA mutation. Diagnosis of congenital thrombotic thrombocytopenic purpura was confirmed by severely deficient ADAMTS13 activity $(\leq 10 \%$ of normal) in the absence of a functional inhibitor and the presence of ADAMTS13 mutations on both alleles. By the end of 2017, 123 confirmed patients had been enrolled from Europe $(n=55)$, Asia $(n=52,90 \%$ from Japan), the Americas ( $n=14)$, and Africa $(n=2)$. First recognized disease manifestation occurred from around birth up to the age of 70 years. Of the 98 different ADAMTS13 mutations detected, c.4143_4144dupA (exon 29; p.Glu1382Argfs* 6 ) was the most frequent mutation, present on 60 of 246 alleles. We found a larger proportion of compound heterozygous than homozygous carriers of ADAMTS13 c.4143_4144dupA with overt disease onset at $<3$ months of age ( $50 \%$ vs. $37 \%$ ), despite the fact that ADAMTS13 activity was $<1 \%$ in 18 of 20 homozygous, but in only 8 of 14 compound heterozygous carriers. An evaluation of overt disease onset in all patients with an available sensitive ADAMTS13 activity assay ( $n=97)$ shows that residual ADAMTS13 activity is not the only determinant of age at first disease manifestation. Registered at clinicaltrials.gov identifier NCT01257269.

Ferrata Storti Foundation

Haematologica 2019

Volume 104(10):2107-2116

\section{Correspondence:}

JOHANNA A. KREMER HOVINGA johanna.kremer@insel.ch

Received: January 16, 2019

Accepted: February 20, 2019.

Pre-published: February 21, 2019.

doi:10.3324/haematol.2019.216796

Check the online version for the most updated information on this article, online supplements, and information on authorship \& disclosures: www.haematologica.org/content/104/10/2107

\section{(C)2019 Ferrata Storti Foundation}

Material published in Haematologica is covered by copyright All rights are reserved to the Ferrata Storti Foundation. Use of published material is allowed under the following terms and conditions:

https://creativecommons.org/licenses/by-nc/4.0/legalcode. Copies of published material are allowed for personal or internal use. Sharing published material for non-commercial purposes is subject to the following conditions: https://creativecommons.org/licenses/by-nc/4.0/legalcode, sect. 3. Reproducing and sharing published material for commercial purposes is not allowed without permission in writing from the publisher. 


\section{Introduction}

Hereditary or congenital thrombotic thrombocytopenic purpura (cTTP; also known as Upshaw-Schulman syndrome; OMIM: 274150) is an autosomal recessive inherited thrombotic microangiopathy (TMA), which can manifest with acute life-threatening disease episodes and frequent relapses. ${ }^{1.5}$ The underlying pathophysiology is an absent or severely reduced activity of ADAMTS13 (a disintegrin and metalloproteinase with thrombospondin type 1 repeats, member 13) caused by bi-allelic mutations in the ADAMTS13 gene. . $^{6.9}$ In the absence of functional ADAMTS13, large von Willebrand factor multimeric strings are not cleaved into normal-sized ones. As a result, platelets adhere to these unusually large, extremely adhesive multimers leading to occlusive microvascular thrombosis. The clinical manifestations of acute TTP include the concomitant occurrence of often severe thrombocytopenia, microangiopathic hemolytic anemia, and a variable degree of ischemic organ damage, particularly affecting the brain, heart and kidneys. If left untreated, mortality exceeds $90 \%{ }^{10}$

Congenital TTP (cTTP) is an ultra-rare disease, affecting about one per 1,000,000 persons. ${ }^{5,11}$ Its rarity makes it difficult to unravel what contributes to its complex and varied clinical presentation and course of disease. Adding to this challenge is the large variety of ADAMTS13 gene mutations $(>200)^{11-14}$ that have been identified (missense, nonsense, splice site mutations, and frameshift mutations caused by small deletions and insertions), and new mutations are being continuously revealed. ADAMTS13 gene variants may abolish or impair ADAMTS13 synthesis, secretion or activity. $12,1,1,15$ Some data suggest that the severity of disease in cTTP is related to residual ADAMTS13 activity. ${ }^{13,16}$ In a cohort of 29 cTTP patients, Lotta et al. ${ }^{17}$ found residual ADAMTS13 activity of $<3 \%$ to be associated with an early disease onset ( $<18$ years of age), an annual event rate of $>1$, and a need for prophylactic plasma therapy. Age at overt onset and diagnosis of cTTP shows, in general, a seemingly dichotomous distribution, with approximately half of patients presenting within their first five years of life, and the other half experiencing disease onset in early adulthood, often during a first pregnancy. ${ }^{11,18-21}$ Childhood-onset cTTP often starts in the neonatal period with hematologic features and severe jaundice. ${ }^{1,22}$ Moreover, Schneppenheim et al. ${ }^{23}$ and von Krogh et al. ${ }^{24}$ reported on variable disease courses in cTTP patients homozygous for the ADAMTS13 c.4143_4144dupA mutation, typically having an ADAMTS13 activity $<1 \%$ of normal. Consequently, the question was asked as to whether residual ADAMTS13 activity is really the major determinant of clinical heterogeneity in cTTP. ${ }^{25}$

The Hereditary TTP Registry (clinicaltrials.gov identifier: NCT01257269) started in 2006 as an international open and ambidirectional cohort study for patients with confirmed and suspected cTTP and their family members. ${ }^{26}$ The Registry's overall aim is to document individual clinical courses, treatment requirements, and to identify possible triggers of acute TTP episodes and disease modifiers in relation to the disease-causing ADAMTS13 mutations. The primary aim of this report is to provide the demographic and clinical characterization of the cTTP cohort at enrollment from when the study started until the end of 2017. A second objective was to evaluate residual
ADAMTS13 activity and its relation to apparent disease onset in the whole cohort, with emphasis on carriers of ADAMTS13 c.4143_4144dupA (p.Glu1382Argfs*6) mutation.

\section{Methods}

\section{Eligibility}

The Hereditary TTP Registry enrolls patients from all over the globe. Those eligible for participation are patients with confirmed or suspected cTTP and their family members (for diagnostic criteria see the Online Supplementary Appendix). In the current study, enrollment data from patients with confirmed cTTP were used for evaluation.

\section{Patient recruitment}

Initially, eligible patients were identified through the Bern TTP Registry and the Nara Medical University Registry on thrombotic microangiopathies (TMA), and enrolled through their treating physicians. Later, patients or their physicians approached the Registry directly through the website (www.ttpregistry.net), based on its related publications ${ }^{24,26}$ and contributions at national and international congresses. The Hereditary TTP Registry includes patients from published reports, and patients who have been newly identified. By the end of 2017, around two-thirds of the 123 confirmed CTTP patients had been reported in case reports and case series between 1981 and 2018 (Online Supplementary Appendix $B)$, prior to their enrollment in the Registry.

The Hereditary TTP Registry was approved by the Lead Cantonal Ethics Committee (CEC) in Bern in 2006 (CEC n. 031/06). The protocol was approved by the Institutional Review Boards or Ethics Committees and Institutional Boards responsible, as applicable, at each site. All Registry participants or their legal representatives provided written informed consent before enrollment.

\section{Data collection at enrollment}

Data collection at enrollment is described in the Online Supplementary Appendix.

\section{ADAMTS13 parameters}

In the majority of patients ( $\mathrm{n}=96)$, ADAMTS13 activity assays, functional inhibitors, anti-ADAMTS13 antibodies, as well as molecular analysis of the ADAMTS13 gene, were performed in the Central Hematology Laboratory, Bern University Hospital, University of Bern, Switzerland (50 patients), and in the Department of Blood Transfusion Medicine, Nara Medical University, Japan (46 patients). In the remaining 27 confirmed patients, analyses were performed in other laboratories employing various assays. For two patients, ADAMTS13 activity values were not reported.

ADAMTS13 activity was determined in Bern by the modified FRETS-VWF73 assay [lower limit of quantification (LLO) $1 \%]^{27,28}$ and in Nara by the chromogenic ADAMTS13-act-ELISA (LLO $0.5 \%)^{29}$

Before combining ADAMTS13 activity data for descriptive analysis, we carried out an assay comparison on 41 plasma samples of cTTP patients (Online Supplementary Table S1).

Functional ADAMTS13 inhibitors were assessed by a Bethesdalike assay and reported in Bethesda units $(\mathrm{BU}) / \mathrm{mL}$, a value $\leq 0.4$ $\mathrm{BU} / \mathrm{mL}$ is considered negative. ${ }^{27,30}$ Anti-ADAMTS13 IgG antibodies were assessed by an in-house ELISA, ${ }^{11}$ or a commercially available ELISA (Technoclone $\left.{ }^{\circledR}\right)$ according to the manufacturer's instructions. A patient's sample was defined negative for anti- 
ADAMTS13 IgG antibodies if the titer value was $<25$ for the former method, ${ }^{11}$ and $<15$ arbitrary units $/ \mathrm{mL}$ for the commercially available ELISA (Technoclone $\left.{ }^{\circledR}\right)$ method.

DNA extraction from leukocytes, amplification of all 29 ADAMTS13 exons with flanking intron-exon boundaries and sequencing were performed using standard methods. Amino acids and nucleotides are numbered according to the recommendation of the Human Genome Variation Society. ${ }^{31}$

\section{Overt disease onset and acute episode definitions}

Definition of overt disease onset and acute episode can be found in the Online Supplementary Appendix.

\section{Statistical analysis}

The statistical analysis is described in the Online Supplementary Appendix.

\section{Results}

\section{Demographics, clinical and biochemical characteristics}

During the period from the beginning of the project in 2006 (clinicaltrials.gov identifier NCT01257269) until the end of 2017, 149 patients were assessed for enrollment in the Hereditary TTP Registry; seven patients were excluded as they did not have cTTP. Of the 142 enrolled patients, 19 patients from 19 families were categorized at the end of 2017 as suspected cTTP patients and did not undergo further analysis in this study; the remaining 123 patients from 117 families had confirmed cTTP (Table 1 and Online Supplementary Figure S1). The female to male ratio was 1:1. Fifty-five and 52 patients were enrolled from Europe and Asia, respectively, 14 from the Americas, and 2 from one site on the African continent (Online Supplementary Figure S2). Accordingly, 65 participants were Caucasian, 52 Asian, and 3 Hispanic. Median age at enrollment was 26.1 years (range: 0.1-75.0 years), median age at clinical diagnosis was 16.7 years (range: 0-69.8 years), and median age at reported overt disease onset was 4.5 years (range: 0-69.8 years). Figure 1 shows the relationship between overt disease onset, clinical diagnosis, and confirmation of the diagnosis for each patient over time.

ADAMTS13 activity values were reported for 121 of 123 patients and $\leq 10 \%$ in 121/121 (Table 1 ). Despite missing ADAMTS13 activity values, two patients were diagnosed with CTTP based on the molecular analysis of the ADAMTS13 gene showing two disease-causing mutations.

ADAMTS13 functional inhibitors were negative in all reported cases, but 12 of 103 patients analyzed had a positive anti-ADAMTS13 antibody result by ELISA (Table 1). Consanguinity of the parents was acknowledged in 14 out of 104 cTTP patients for whom this information was provided. Forty-seven patients were homozygous and 76 compound heterozygous mutation carriers. In total, 98 different ADAMTS13 mutations were identified in 123 confirmed cTTP patients.

\section{Occurrence of diseases and disorders up until enrollment}

Information on the occurrence of concomitant diseases and disorders up until enrollment was available for 120 cTTP patients (Table 2). Jaundice (due to hemolysis or liver disease, $49 \%$ ) and arterial thromboembolic events
Table 1. Demographic and clinical features and ADAMTS13-related laboratory findings in congenital thrombotic thrombocytopenic purpura patients at enrollment.

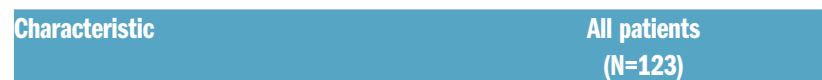
Median age at enrollment $(\mathrm{n}=118)^{\dagger}$
$26.1[0.1,15.1,37.2,75.0]$

Median age at overt disease onset $(\mathrm{n}=111) \quad 4.52[0.00,0.01,20.1,69.8]$

Median age at clinical diagnosis $(\mathrm{n}=122) \quad 16.7[0.00,4.00,28.6,69.8]$

Gender (F/M) 62/61

Ethnicity (self-reported)

\begin{tabular}{lc} 
Caucasian & $65(53 \%)$ \\
Hispanic & $3(2.4 \%)$ \\
Asian & $52(42 \%)$ \\
Other & $3(2.4 \%)$ \\
ADAMTS13 activity (s10\%) & $121 / 121$ \\
\hline ADAMTS13 functional inhibitor $(+)$ & $0 / 114$ \\
Anti-ADAMTS13 antibodies $(+)^{\S}$ & $12 / 103$ \\
\hline Consanguinity of parents & $14 / 104$ \\
Homozygous genotypes & $47 / 123$ \\
\hline Compound heterozygous genotypes & $76 / 123$ \\
\hline
\end{tabular}

$\mathrm{N}$ : total number of patients; $\mathrm{n}$ : number of patients with available values; $\mathrm{F}$ : female; $\mathrm{M}$ male. Continuous variables are presented as median (minimum, $25^{\text {th }}$ percentile, $75^{\text {th }}$ percentile, maximum). Categorical variables are presented as number and percentage of all patients, or as number of $n$ patients. Five patients from five families were enrolled postmortem by consent of their family members. ${ }^{\circledR} \mathrm{A}$ patient's sample was defined positive for Anti-ADAMTS13 IgG antibodies if the titer value was $>25$ for the inhouse ELISA method, ${ }^{11}$ and $>15$ arbitrary units $/ \mathrm{mL}$ for the commercially available ELISA (Technoclone $\left.{ }^{\circledR}\right)$ method.

$(28 \%)$ were frequently reported. Stroke had occurred in $21 \%$, and transient ischemic attack in $10 \%$. Arterial thromboembolic events had occurred in all age groups. In the groups $>40-50$ years and $>50$ years, $50 \%$ or more of the cTTP patients had suffered from at least one arterial thromboembolic event (Figure 2). Other neurological disorders included epileptic seizures (6 patients), headache (5 patients), and psychiatric conditions (depression, behavioral and mental disorders; 20 patients).

Renal insufficiency occurred in $25 \%$ of the cohort. Twelve patients needed hemodialysis and 3 underwent a kidney transplantation. Autoimmune disorders (hypothyroidism, IgA nephritis, hyperthyroidism) were rare. Finally, transfusion-transmitted viral diseases were reported in 13 of 120 patients (11\%).

\section{Reported acute episodes and their context}

A total of 291 distinct acute TTP episodes were recorded in 81 patients (Online Supplementary Table S2), while there was no detailed information on acute episodes in 42 patients. Patients had experienced a median of 2 episodes (range: 1-22) prior to enrollment, corresponding to a median of 0.10 (range: 0.02-8.91) acute episodes per year. Median duration of an episode was seven days (range: < 1-128 days).

For 287 of the 291 episodes, information on possible triggers of the acute episode was available; in 190 of 287 episodes one or more trigger(s) was/were presumed by the treating physicians (Figure 3). Infection was the most frequent trigger reported ( $41 \%$ of episodes). Alcohol excess was the trigger for 30 episodes and was observed only in male patients, while 28 episodes occurred during 


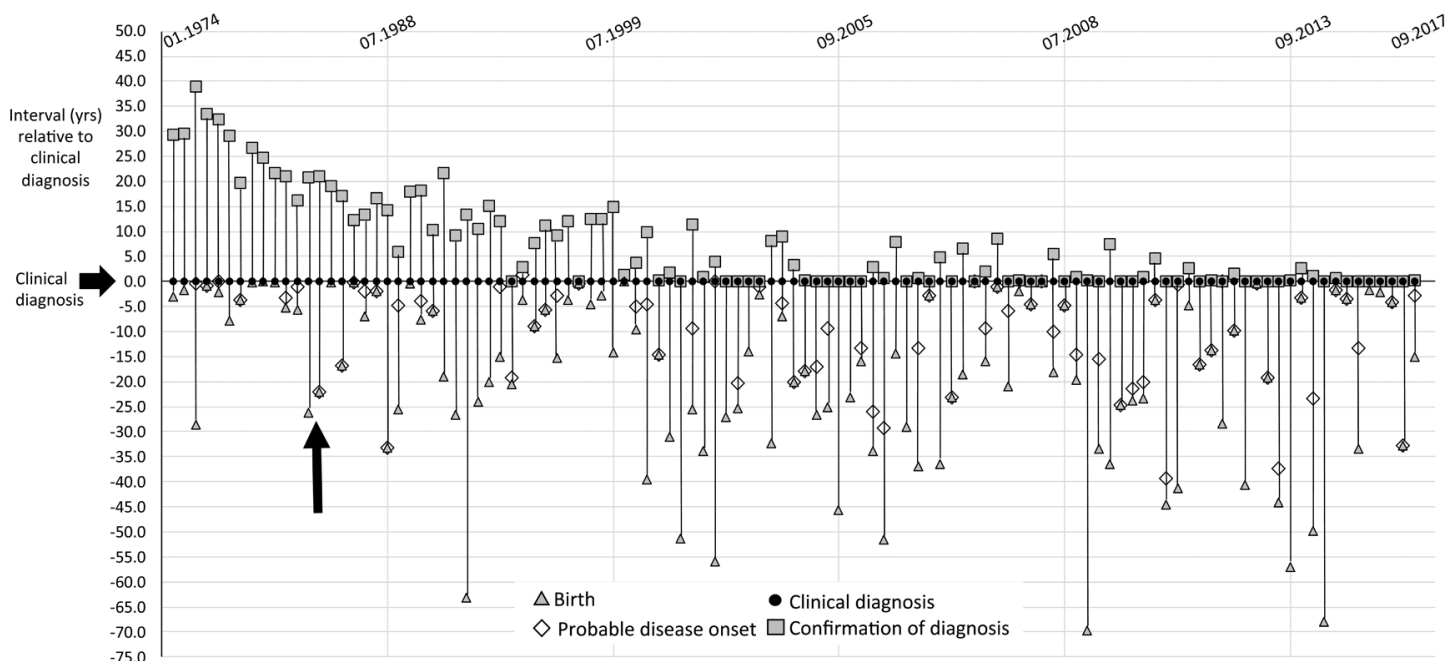

Figure 1. Clinical diagnosis and confirmation of diagnosis in relation to overt disease onset: information available for 111 confirmed congenital thrombotic thrombocytopenic purpura (cTTP) patients. $x$-axis: each patient's individual time point of clinical diagnosis is shown. Below the x-axis, the interval (in years, yrs) between birth, (probable) disease onset, and clinical diagnosis is shown. Above the x-axis, the interval (in yrs) between clinical diagnosis and confirmation of diagnosis is shown. Disease onset varied from as early as the new-born period up to 70 years of age. The earliest clinical diagnosis of TTP in a Registry patient was put forward in January 1974. The patient highlighted by a vertical arrow was born 22 years before the clinical diagnosis of cTTP was established although disease onset was documented in the neonatal period. Confirmation of the CTTP diagnosis was achieved by means of ADAMTS13 testing 21 years after the clinical diagnosis. NB: ADAMTS13 was first described in 1996, ADAMTS13 assays became more widely available around the turn of the millennium.

pregnancy. In addition, drug/medication use was assumed as trigger for 13 episodes, and 18 episodes seemed to be triggered by the patient's birth, injury, food poisoning, and various others.

\section{Prophylactic treatment}

Seventy-one percent of the 117 cTTP patients with available information received regular treatment, predominantly with plasma products (99\%) (Table 3). Plasma products used included fresh frozen plasma in 68 patients, two received fresh frozen plasma and cryo-poor plasma, and 12 patients received solvent/detergent plasma. One patient received a plasma-derived FVIII product (Koate $\left.{ }^{\circledR}\right)$. The median interval of the regular treatments was 14.0 days (range: 2-75 days). Thirty-four patients were treated on demand. These data represent a snap-shot at the time of enrollment, whereby regular treatment was intensified or reduced depending on the patient's individual requirements.

\section{ADAMTS13 mutations}

In the 123 confirmed cTTP patients, 98 different ADAMTS13 mutations were identified in 245 alleles (Online Supplementary Table S3). There were 57 missense, 12 nonsense, 21 frameshift due to deletions or insertions, and 8 splice site mutations. In one patient, only one mutation was detected and diagnosis of cTTP was confirmed through a plasma infusion trial. The most frequent mutation observed was ADAMTS13 c.4143_4144dupA (present on 60 of 246 alleles) followed by c. $3178 \mathrm{C}>\mathrm{T}$ (13 of 246 alleles) and c.577C>T (11 of 246 alleles) (Online Supplementary Figure S4). In addition, there were three mutations found in six of 246 alleles $(2.4 \%)$, one mutation in 5 of 246 alleles $(2.0 \%), 4$ mutations in four of 246 alleles
(1.6\%), six mutations in 3 of 246 alleles (1.2\%), and 81 mutations were found only once $(n=58)$ or twice $(n=23)$. The mutation c.3650T $>C$ was the only mutation found on three different continents: the Americas, Asia, and Europe, involving 4, 1, and 1 alleles, respectively. Mutation c.4143_4144dupA was found on one and 59 alleles, and c. $3178 \mathrm{C}>\mathrm{T}$ on 3 and 10 alleles in the Americas and Europe, respectively. Mutation c.3616C $>\mathrm{T}$ was found with one allele in Europe and two alleles in Asia, and mutation c.4006C > T was found in both Europe and Asia with two alleles each. All other mutations were restricted to one single continent each. In Asia, c.577C $>\mathrm{T}$ is the most frequent mutation, found exclusively in Japan (11 alleles) (Online Supplementary Table S3).

Nineteen of the 98 mutations have not been reported before. Mutations were found across the ADAMTS13 gene and in all protein domains without indication for a genetic hot-spot.

\section{Characteristics, overt disease onset and ADAMTS13 activity in homozygous and compound heterozygous carriers of ADAMTS13 4143_4144dupA mutation}

Carriers of the ADAMTS13 c.4143_4144dupA (p.Glu1382Argfs*6) mutation in exon 29 were enrolled from sites in Austria, Czech Republic, Hungary, Norway, Poland, and the USA. The number of homozygous and compound heterozygous carriers was about equal $(P=0.51)$. Age at first diagnosis and at enrollment tended to be lower in compound heterozygous compared to homozygous carriers ( $P=0.06$ and $P=0.08$, respectively) (Table 4). Information on recognized overt disease onset was available for 14 compound heterozygotes and 19 homozygotes. Homozygotes had a numerically higher age at overt onset (5.0 years; range $0-22.3$ years) than com- 


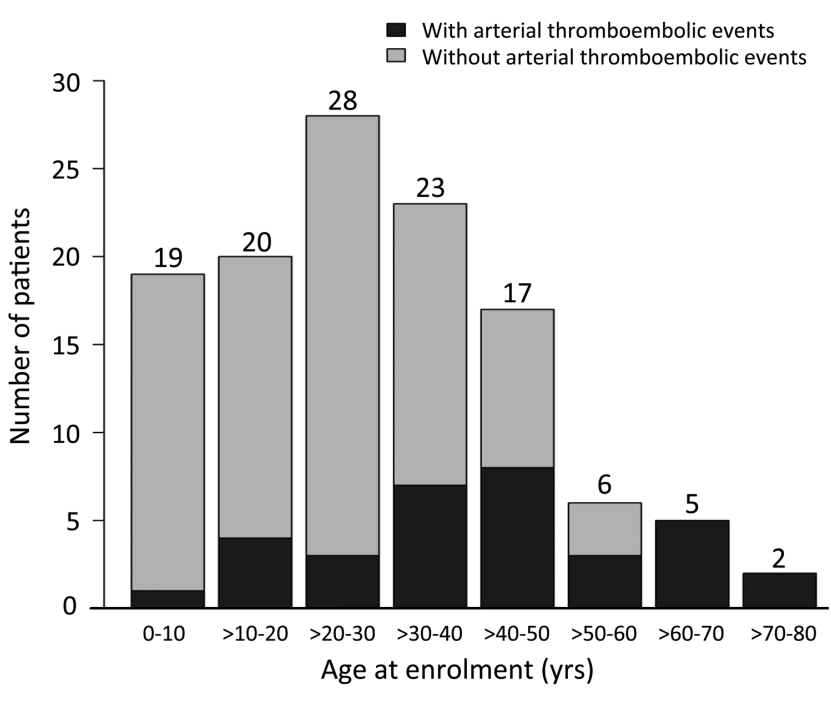

Figure 2. Number of congenital thrombotic thrombocytopenic purpura (cTTP) patients with or without a history of arterial thromboembolic events by age category at enrollment. Data are available for 120 of 123 confirmed cTTP patients. For five deceased patients, age at death was used. Yrs: years.

pound heterozygotes (1.1 years; $0-35.0$ years), however, the difference was not significant $(P=0.6)$, nor were the differences of all the other clinical characteristics (Table 4). Reported onset in homozygous ADAMTS13 c.4143_4144dupA carriers has a bimodal pattern with peaks in the neonatal period and between six years and $<18$ years of age, whereas in compound heterozygotes the proportion of patients with later overt onset steadily declines (Online Supplementary Figure S3A).

In 34 of 38 ADAMTS13 c.4143_4144dupA carriers, ADAMTS13 activity was assessed by the modified FRETS-VWF73 assay at the Central Hematology Laboratory, Bern University Hospital, Bern, Switzerland. ADAMTS13 activity was $<1 \%$ in 18 of 20 (90\%) homozygous and in 8 of $14(57 \%)$ compound heterozygous carriers (Online Supplementary Figure S3B). The second ADAMTS13 mutation in these eight patients was: c.1520G >A (p.Arg507Glu) in 5 alleles; c.1313G>T (p.Cys438Phe), c.2455delG (p.Ala819Leufs*24), and c.4091_4092insA (p.His1364Glnfs*) each in one allele. In the five compound heterozygotes with an ADAMTS13 activity of $1 \%$ to $<5 \%$, we found the following five mutations: c.1313G $>\mathrm{T}$ (p.Cys438Phe), c.2410T $>\mathrm{C}$ (p.Cys804Arg), c.2836T >C (p.Cys946Arg), c.3178C>T (p.Arg1060Trp) and c.3650T >C (p.Ile1217Thr). c.3178C>T (p.Arg1060Trp) was also present in the compound heterozygous carrier with an ADAMTS13 activity of $6.3 \%$. In c.4143_4144dupA carriers having an ADAMTS13 activity $<1 \%$, information on disease onset was available in 15 homozygotes and seven compound heterozygotes, with a trend for later disease onset in homozygotes (Online Supplementary Figure S3C).

\section{Overt disease onset and ADAMTS13 activity in all congenital thrombotic thrombocytopenic purpura patients}

Reported overt disease onset in 97 cTTP patients whose ADAMTS13 activity was measured either in Bern, Switzerland, or Nara, Japan, is shown in relation to the
Table 2. Reported concomitant diseases and disorders in congenital thrombotic thrombocytopenic purpura patients up to enrollment.

\begin{tabular}{lc}
\hline IIype of disease/disorder & $\begin{array}{c}\text { All patients } \\
\text { (N=120) }\end{array}$ \\
Arterial thromboembolic diseases $^{\dagger}$ & $33(28 \%)$ \\
Myocardial infarction & $5(4.2 \%)$ \\
Transient ischemic attack & $12(10 \%)$ \\
Stroke & $25(21 \%)$ \\
Other & $6(5.0 \%)$ \\
Other neurological disorders ${ }^{\dagger}$ & $27(22 \%)$ \\
Epileptic seizure & $6(5.0 \%)$ \\
Headache & $5(4.2 \%)$ \\
Various other & $20(17 \%)$ \\
\hline Renal insufficiency & $30(25 \%)$ \\
Hemodialysis & $12(10 \%)$ \\
Kidney transplant & $3(2.5 \%)$ \\
Jaundice (hemolysis or liver disease) & $59(49 \%)$ \\
Hyperbilirubinemia in neonatal period ${ }^{\dagger}$ & $30(25 \%)$ \\
\hline Anemia & \\
Iron deficiency & $8(6.7 \%)$ \\
Renal anemia & $8(6.7 \%)$ \\
Other diseases & \\
Cancer & $2(1.7 \%)$ \\
Autoimmune disorders & $4(3.3 \%)$ \\
Transfusion-transmitted viral infection ${ }^{\dagger}$ & $13(11 \%)$ \\
HBV & $3(2.5 \%)$ \\
\hline HCV & $10(8.3 \%)$ \\
\hline
\end{tabular}

$\mathrm{N}$ : total number of patients with information available; HBV: hepatitis B viral infection HCV: hepatitis C viral infection; HIV: human immunodeficiency virus infection Categorical variables are presented as numbers and percentage of all patients. 'For different disease categories the total number of affected patients is given (as a patient may have incurred more than one disorder per category, the sum of all conditions per category may be larger than that of the number of affected patients). "No information reported for seven patients on hyperbilirubinemia in the neonatal period.

measured ADAMTS13 activity (Figure 4). Twenty-eight of 70 patients with ADAMTS13 activity $<1 \%$ had overt disease onset in the neonatal period, but there were also 20 patients with overt disease onset at age $>15-57$ years. Most patients with measurable ADAMTS13 activity ( $\geq$ $1 \%$ ) had a reported disease onset at $>5$ years of age. Overall, we found a weak but significant correlation between age at overt onset and ADAMTS13 activity $\left(r_{s}=0.25, P<0.01\right)$.

\section{Discussion}

We report the successful implementation of an International Registry for the ultra-rare disease cTTP. After many years of preparation, patient recruitment and enrollment, by the end of 2017, a total of 123 confirmed cTTP patients from 117 families were included in the study by centers from all over the world with the help of numerous primary care physicians (see the complete list in Online Supplementary Appendix A).

Congenital TTP is often not recognized at the first occurrence of disease manifestation, e.g. in the newborn 
period or during childhood..$^{8,11,32,33}$ As demonstrated for the 111 patients with available information, a substantial number of affected subjects showed thrombocytopenia, hemolysis, jaundice, petechiae and/or anemia during the neonatal period, often leading to therapeutic interventions such as exchange blood transfusion, sometimes many years before clinical diagnosis of TTP (Figure 1). Other patients, however, seemed to present the first signs of TTP only in adulthood. Before the late 1990s cTTP was purely a clinical diagnosis. Today, clarification of its pathophysiology, $3,34-36$ the advent of ADAMTS13 assays, and the possibility of sequencing the ADAMTS13 gene emerging over the past two decades ${ }^{6,7,9,11,12,35-38}$ allows the diagnosis of both autoimmune and congenital TTP to be quickly and efficiently confirmed (for consensus definitions of diagnosis see Scully et al. ${ }^{4,22}$ and Kremer Hovinga et al. ${ }^{5}$ ). During recent years, we have been able to confirm the clinical diagnosis of many of our patients within days or weeks through the use of appropriate assays (Figure 1).

The systematic collection of clinical data in individual patients revealed substantial comorbidities in cTTP patients (Table 2 and Figure 2). Most notable is the high proportion of patients who had suffered from premature arterial thromboembolic events, mainly transient ischemic attacks, ischemic strokes and, to a lesser extent, myocardial infarctions. At 40-50 years of age and over, more than $50 \%$ of cTTP patients had suffered from at least one arte- rial thromboembolic event. In addition, several patients seem to suffer from headache, mental or depressive disorders, which is similar to the situation in acquired TTP. ${ }^{39,40}$ Of note, $25 \%$ of cTTP patients had renal insufficiency at the time of enrollment and $12.5 \%$ had required hemodialysis or underwent kidney transplantation (Table 2). Severe and, sometimes transient, renal failure has been described in case reports or series of cTTP patients, ${ }^{41-43}$ which is distinct from acquired TTP where a creatinine $\geq 2 \mathrm{mg} / \mathrm{dL}$ $(\geq 180 \mu \mathrm{mol} / \mathrm{L})$ is a clear exception. ${ }^{5,22,44}$

Detailed information on 291 acute episodes in 81 cTTP patients was available at the time of enrollment. The frequency of acute manifestations varied considerably in individual patients with a median of approximately 0.1 acute episode per patient-year and a range of 0.02-9 yearly acute episodes per patient (Online Supplementary Table S2). In two-thirds of episodes, the responsible physicians assumed a triggering factor, mainly mild to moderately severe infections, alcohol excess in men, pregnancy in women, and various other potential triggers (Figure 3). Pregnancy is a well-known trigger for acute disease episodes in cTTP $13,18-20,45$ and it is conceivable that pregnancy and other conditions leading to an increased endothelial von Willebrand factor secretion may bring about acute disease in the face of absent or severely reduced ADAMTS13 activity. It is generally thought that an acute episode in cTTP is rapidly controlled by simple plasma

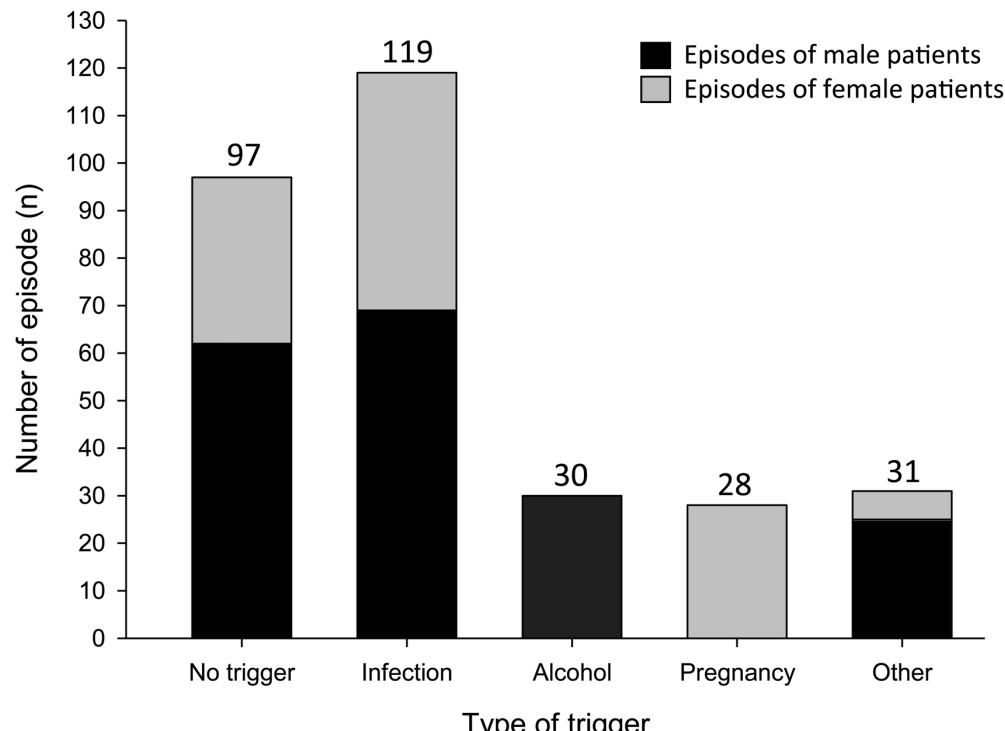

Figure 3. Triggers of acute thrombotic thrombocytopenic purpura (TTP) episodes until enrollment in male and female congenital thrombotic thrombocytopenic purpura patients. For 287 of 291 acute TTP episodes documented in the Hereditary TTP Registry information on triggers of the acute bout was known. Seventy-nine patients had episodes with or without presumed triggers: for 97 episodes in 46 patients no trigger was reported; for 190 episodes in 58 patients triggers were reported. Triggers do not sum up as a patient may have had more than one trigger of an acute episode, nor do patients as they may have had episodes with or without triggers.

Table 3. Reported treatment in congenital thrombotic thrombocytopenic purpura patients at enrollment.

$\begin{array}{lc}\text { Tarialiles } & \begin{array}{c}\text { All patients } \\ \left(\mathbf{N}=\mathbf{1 1}^{*}\right.\end{array} \\ \text { Mode of treatment } & 83(71 \%) \\ \text { Patients with regular prophylactic treatment } & 34(29 \%) \\ \text { Patients with on demand treatment } & 82(99 \%) \\ \text { Plasma and Factor products applied among regularly treated patients } & 1(1.2 \%) \\ \text { Plasma products }{ }^{\dagger} & 14.0[2.0,14.0,21.0,75.0] \\ \text { Plasma derived FVIII product } & \end{array}$

$\mathrm{N}$ : total number of patients; $\mathrm{n}$ : number of patients with available values. Continuous variables are presented as median [(minimum, $25^{\text {th }}$ percentile, $75^{\text {th }}$ percentile, maximum) Categorical variables are presented as number and percentage of all patients, or as number (n) of patients. *Information on current treatment missing for two male and four female patients. 'Fresh frozen plasma ( $\mathrm{n}=68)$; fresh frozen plasma and cryo-poor plasma ( $\mathrm{n}=2$ ), solvent/detergent plasma ( $\mathrm{n}=12)$. ${ }^{\dagger 1} \mathrm{Interval}$ missing for five patients. 
infusion; ${ }^{2,18}$ however, data from our Registry show that the median duration of over 200 well-documented episodes was seven days, probably because of delayed plasma therapy or illness from the triggering event.

Information on prophylactic treatment was available for 117 of 123 cTTP patients (Table 3). Twenty-nine percent of the patients had no regular prophylaxis and received plasma only on demand. Seventy-one percent were on prophylactic treatment, usually with fresh frozen plasma or solvent/detergent plasma infusions, while only one patient was treated with a commercial Factor VIII concentrate (Koate $\left.{ }^{\circledR}\right)$. Dosing intervals mostly varied between two and three weeks, as had been proposed earlier on the basis of platelet count measurements. ${ }^{18}$ Whether such a dosing interval sufficiently controls minor TTP-related symptoms is questionable given the frequently reported

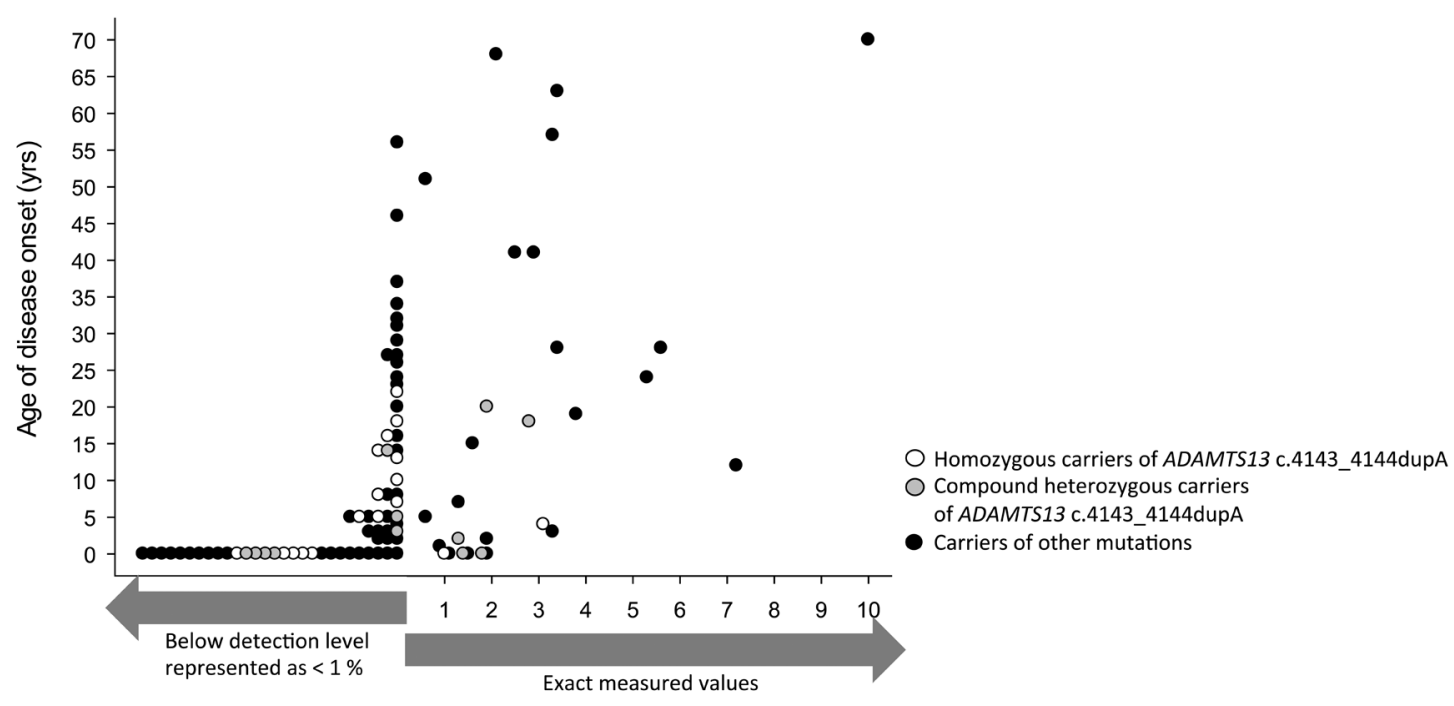

ADAMTS13 activity (\%)

Figure 4. Age at disease onset in relation to ADAMTS13 activity in 97 congenital thrombotic thrombocytopenic purpura (cTTP) patients. Ninety-seven confirmed CTTP patients had information on disease onset and an ADAMTS13 activity determination performed either at the Central Hematology Laboratory, Bern University Hospital, Switzerland, or at the Department of Blood Transfusion Medicine, Nara Medical University, Japan. Patients are labeled according to their ADAMTS13 mutations: 17 homozygous carriers and 12 compound heterozygous carriers of ADAMTS13 c.4143_4144dupA are shown with white and gray circles, respectively. Black circles represent carriers of other mutations. Spearman's rank correlation coefficient for age at overt disease onset versus ADAMTS13 activity: $r_{\mathrm{s}}=0.25, P<0.01$. yrs: years.

Table 4. Characteristics of congenital thrombotic thrombocytopenic purpura patients carrying one or two ADAMTS13 c.4143_4144dupA alleles.

\begin{tabular}{|c|c|c|c|}
\hline \multirow[b]{2}{*}{ Variables } & \multicolumn{2}{|c|}{ Genotype } & \multirow[b]{2}{*}{$\mathbf{P}$} \\
\hline & $\begin{array}{l}\text { Homozygous } \\
\qquad(\mathrm{N}=22)\end{array}$ & $\begin{array}{l}\text { Compound heterozygous } \\
\qquad(\mathbb{N}=16)\end{array}$ & \\
\hline Median age of patient at enrollment (yrs) & $31.2[17.0,60.1]$ & $23.2[4.0,60.1]$ & 0.08 \\
\hline Median age of patient at clinical diagnosis (yrs) & $20.4[1.8,51.5]$ & $4.35[0.2,39.6]$ & 0.06 \\
\hline Female sex & $8(36 \%)$ & $9(56 \%)$ & 0.32 \\
\hline \multicolumn{4}{|l|}{ Occurrence of major diseases/disorders } \\
\hline Transient ischemic attack & $4(18 \%)$ & $4(25 \%)$ & 0.70 \\
\hline Stroke & $5(23 \%)$ & $4(25 \%)$ & 1.00 \\
\hline (Chronic) renal insufficiency & $7(32 \%)$ & $6(38 \%)$ & 0.72 \\
\hline Jaundice (hemolysis or liver disease) & $5(23 \%)$ & $2(13 \%)$ & 0.68 \\
\hline Diagnosis of a transfusion transmitted viral disease & $1(4.5 \%)$ & $0(0 \%)$ & 1.00 \\
\hline \multicolumn{4}{|l|}{ Mode of treatment } \\
\hline Number of patients with regular prophylactic treatment & $17(77 \%)$ & $11(69 \%)$ & 0.71 \\
\hline Median interval of most recent treatment (days)* & $21.0[7.00,75.0]$ & $14.0[14.0,56.0]$ & 0.19 \\
\hline \multicolumn{4}{|l|}{ Reported episodes } \\
\hline Number of patients with reported episodes prior to enrollment & $15(68 \%)$ & $11(69 \%)$ & 1.00 \\
\hline Median number of episodes per patient & $6.00[1.00,11.0]$ & $1.00[1.00,12.0]$ & 0.13 \\
\hline Number of retrospective episodes per year & $0.15[0.03,0.35]$ & $0.12[0.03,0.80]$ & 0.62 \\
\hline
\end{tabular}

$\mathrm{N}$ : total number of patients. Continuous variables are presented as median (minimum, maximum). Categorical variables are presented as number and percentage of all patients. *Interval missing for two homozygous patients. yrs: years 
symptoms like headache or mental disorders. Even though ADAMTS13 half-life in plasma is about 2-4 days ${ }^{46,47}$ almost all patients will have unmeasurable ADAMTS13 levels within 7-10 days after infusion of 2-4 units of fresh frozen plasma. Evidently, a prospective follow up of cTTP patients will be needed to optimize replacement therapy. In the near future, the availability of rhADAMTS13, successfully tested in a pharmacokinetics and safety study in 15 cTTP patients, ${ }^{48}$ will greatly facilitate regular prophylactic treatment, and will probably allow treatment to be carried forward at home. In addition, given the high risk of acute episodes, ischemic strokes and other sequelae, a general prophylactic ADAMTS13 replacement in cTTP may be indicated.

ADAMTS13 mutations were found in all except one cTTP Bi-allelic patient (Online Supplementary Table S3 and Online Supplementary Figure S4); a total of 98 different mutations spread throughout the ADAMTS13 gene were identified, including several mutations that have not so far been described. Eighty-one mutations occurred only once (58 alleles) or twice, 14 different mutations were identified on 3-6 alleles, whereas three variants were present on 11 (c.577C>T), 13 (c.3178C>T), and 60 (c.4143_4144dupA) alleles, respectively. The latter three recurring mutations have been repeatedly found in Japanese (c.577C $>\mathrm{T})^{11,37}$ and Caucasian (c.3178C $>\mathrm{T}$ and c.4143_4144dupA) patients. ${ }^{15,20,23,24,45,49}$ It has been postulated that mutations leading to a completely absent ADAMTS13 activity show a more severe phenotype than those associated with some residual ADAMTS13 activity. ${ }^{13,16,17,49}$ We compared disease characteristics of 22 homozygous and 16 compound heterozygous carriers of the c.4143_4144dupA mutation (Table 4 and Online Supplementary Figure S3A-C). Median age at diagnosis tended to be higher in homozygous carriers, even though there was a wide range of age at diagnosis from 0.2-51.5 years in both groups. There was no difference in any other of the disease characteristics. Despite a severely deficient ADAMTS13 activity $(<0.5-1 \%$ of normal) in homozygous c.4143_4144dupA carriers, almost half of affected patients had an overt disease onset at an age of $>6$ years that seemed to be higher than in compound heterozygotes with equally severe ADAMTS13 deficiency (Online Supplementary Figure S3C). Shang et al. ${ }^{50}$ suggested that the c.4143_4144dupA mutation might be associated with defective apical, but preserved basolateral, secretion by endothelial cells. Whether some ADAMTS13 activity at the endothelial cell layer in the absence of plasmatic ADAMTS13 activity is present that may explain a delayed TTP onset in a proportion of homozygous carriers needs further study.

We then tested whether overt disease onset was related with residual plasma ADAMTS13 activity in all patients whose ADAMTS13 activity was measured in Nara or Bern after a comparative evaluation had shown a good agreement of activity levels in the very low range below $5-10 \%$ (Online Supplementary Table S1). For 97 patients with available information, it was evident that overt disease onset was not strictly related to residual ADAMTS13 activity. Whereas about $40 \%$ of cTTP patients with an ADAMTS13 activity $<1 \%$ had a neonatal disease onset, $20 \%$ seemed to have a first TTP manifestation at age $>20$ years. On the other hand, most cTTP patients with an ADAMTS13 activity $>1-2 \%$ had a delayed apparent disease manifestation (Figure 4). Thus, residual ADAMTS13 activity is clearly not the only determinant of disease severity, as reflected by age at overt onset.

In conclusion, the Hereditary TTP Registry has provided substantial information, but it will be of utmost importance for the development of an optimized management strategy to enlarge the cohort by directly enrolling more patients from around the globe, and through embarking on collaborations with other established registries on cTTP. It would be highly desirable to prospectively follow all cTTP patients at regular intervals and evaluate the best prophylactic strategy to avoid unnecessary deaths and late sequelae in this treatable condition.

A complete list of the collaborators of the Hereditary TTP Registry appears in the Online Supplementary Appendix A.

\section{Acknowledgments}

Acknowledgments to the late professor Miha Furlan for his pioneering work on discovery of von Willebrand factor-cleaving protease ADAMTS13 and its deficiency in TTP more than 20 years ago. The authors thank the teams of the laboratories in Bern, Switzerland and Nara, Japan for work on ADAMTS13related assays and molecular analyses of the ADAMTS13 gene. Corinne Eschler is thanked for her assistance in data collection and entry.

\section{Funding}

The hereditary TTP registry has received support through grants from the Swiss National Science Foundation (Grant 32003B-124892), the Mach-Gaensslen Foundation Switzerland, the ISTH 2007 Presidential Fund, the NFG Foundation, as well as research grants from Baxalta US Inc., member of the Takeda group of companies, Bannockburn, IL, USA (IIR Grant no H10-000600 and H16-36165). Furthermore, this work was supported in part by grants-in-aid from the Ministry of Health, Labor, and Welfare of Japan, and Takeda Science Foundation (to YF and MM), and National Heart, Lung, and Blood Institute of the National Institutes of Health under Award Number 1K01HL135466 (to DRT).

\section{References}

1. Upshaw JD Jr. Congenital deficiency of a factor in normal plasma that reverses microangiopathic hemolysis and thrombocytopenia. N Engl J Med. 1978;298(24):1350-1352.

2. Kinoshita S, Yoshioka A, Park YD, et al. Upshaw-Schulman syndrome revisited: a concept of congenital thrombotic thrombocytopenic purpura. Int J Hematol. 2001;74(1): 101-108.

3. Moake JL. Thrombotic microangiopathies. N Engl J Med. 2002;347(8):589-600.
4. Scully M, Cataland S, Coppo P, et al Consensus on the standardization of terminology in thrombotic thrombocytopenic purpura and related thrombotic microangiopathies. J Thromb Haemost. 2017; 15(2):312-322.

5. Kremer Hovinga JA, Coppo P, Lämmle B, Moake JL, Miyata T, Vanhoorelbeke K. Thrombotic thrombocytopenic purpura. Nat Rev Dis Primers. 2017;3:17020.

6. Levy GG, Nichols WC, Lian EC, et al. Mutations in a member of the ADAMTS gene family cause thrombotic thrombocy- topenic purpura. Nature. 2001; 413(6855): 488-494.

7. Kokame K, Matsumoto M, Soejima K, et al. Mutations and common polymorphisms in ADAMTS13 gene responsible for von Willebrand factor-cleaving protease activity. Proc Natl Acad Sci U S A. 2002;99(18):1190211907.

8. Schneppenheim R, Budde U, Oyen F, et al von Willebrand factor cleaving protease and ADAMTS13 mutations in childhood TTP. Blood. 2003;101(5):1845-1850.

9. Veyradier A, Lavergne JM, Ribba AS, et al. 
Ten candidate ADAMTS13 mutations in six French families with congenital thrombotic thrombocytopenic purpura (UpshawSchulman syndrome). J Thromb Haemost. 2004;2(3):424-429.

10. Amorosi EL, Ultmann JE. Thrombotic thrombocytopenia purpura: Report of 16 cases and review of the literature. Medicine. 1966;45 (2):139-160.

11. Fujimura $Y$, Matsumoto $M$, Isonishi $A$, et al. Natural history of Upshaw-Schulman syndrome based on ADAMTS13 gene analysis in Japan. J Thromb Haemost. 2011;9 Suppl 1:283-301.

12. Lotta LA, Garagiola I, Palla R, Cairo A, Peyvandi F. ADAMTS13 mutations and polymorphisms in congenital thrombotic thrombocytopenic purpura. Hum Mutat. 2010;31(1):11-19.

13. Camilleri RS, Scully M, Thomas M, et al. A phenotype-genotype correlation of ADAMTS13 mutations in congenital thrombotic thrombocytopenic purpura patients treated in the United Kingdom. J Thromb Haemost. 2012;10(9):1792-1801.

14. Joly BS, Boisseau P, Roose E, et al. ADAMTS13 Gene Mutations Influence ADAMTS13 Conformation and Disease AgeOnset in the French Cohort of UpshawSchulman Syndrome. Thromb Haemost. 2018;118(11):1902-1917.

15. Pimanda JE, Maekawa A, Wind T, Paxton J, Chesterman CN, Hogg PJ. Congenital thrombotic thrombocytopenic purpura in association with a mutation in the second CUB domain of ADAMTS13. Blood. 2004;103(2): 627-629.

16. Lotta LA, Wu HM, Musallam KM, Peyvandi F. The emerging concept of residual ADAMTS13 activity in ADAMTS13-deficient thrombotic thrombocytopenic purpura. Blood Rev. 2013;27(2):71-76.

17. Lotta LA, Wu HM, Mackie IJ, et al. Residual plasmatic activity of ADAMTS13 is correlated with phenotype severity in congenital thrombotic thrombocytopenic purpura. Blood. 2012;120(2):440-448.

18. Furlan M, Lämmle B. Aetiology and pathogenesis of thrombotic thrombocytopenic purpura and haemolytic uraemic syndrome: the role of von Willebrand factor-cleaving protease. Best Pract Res Clin Haematol. 2001;14(2):437-454

19. Fujimura $Y$, Matsumoto M, Kokame K, et al. Pregnancy-induced thrombocytopenia and TTP, and the risk of fetal death, in UpshawSchulman syndrome: a series of 15 pregnancies in 9 genotyped patients. $\mathrm{Br} \mathrm{J}$ Haematol. 2009;144(5):742-754

20. Moatti-Cohen M, Garrec C, Wolf M, et al. Unexpected frequency of Upshaw-Schulman syndrome in pregnancy-onset thrombotic thrombocytopenic purpura. Blood. 2012;119(24):5888-5897.

21. Scully $M$, Thomas $M$, Underwood $M$, et al. Thrombotic thrombocytopenic purpura and pregnancy: presentation, management, and subsequent pregnancy outcomes. Blood. 2014;124(2):211-219.

22. Scully M, Hunt BJ, Benjamin S, et al. Guidelines on the diagnosis and management of thrombotic thrombocytopenic purpura and other thrombotic microangiopathies. Br J Haematol. 2012;158(3):323-335

23. Schneppenheim R, Kremer Hovinga JA, Becker $\mathrm{T}$, et al. A common origin of the 4143insA ADAMTS13 mutation. Thromb Haemost. 2006;96(1):3-6.

24. von Krogh AS, Quist-Paulsen P, Waage A, et al. High prevalence of hereditary thrombotic thrombocytopenic purpura in central Norway: from clinical observation to evidence. J Thromb Haemost. 2016;14(1):73-82.

25. Kremer Hovinga JA, Heeb SR, Skowronska M, Schaller M. Pathophysiology of thrombotic thrombocytopenic purpura and hemolytic uremic syndrome. J Thromb Haemost. 2018;16(4):618-629

26. Mansouri Taleghani M, von Krogh AS, Fujimura Y, et al. Hereditary thrombotic thrombocytopenic purpura and the hereditary TTP registry. Hamostaseologie. 2013; 33(2):138-143.

27. Kremer Hovinga JA, Vesely SK, Terrell DR, Lämmle B, George JN. Survival and relapse in patients with thrombotic thrombocytopenic purpura. Blood. 2010;115(8):1500-1511

28. Froehlich-Zahnd R, George JN, Vesely SK, et al. Evidence for a role of anti-ADAMTS13 autoantibodies despite normal ADAMTS13 activity in recurrent thrombotic thrombocytopenic purpura. Haematologica. 2012 97(2):297-303.

29. Kato S, Matsumoto M, Matsuyama T, Isonishi A, Hiura H, Fujimura Y. Novel monoclonal antibody-based enzyme immunoassay for determining plasma levels of ADAMTS13 activity. Transfusion. 2006 46(8):1444-1452.

30. Page EE, Kremer Hovinga JA, Terrell DR, Vesely SK, George JN. Thrombotic thrombocytopenic purpura: diagnostic criteria, clinical features, and long-term outcomes from 1995 through 2015. Blood Adv. 2017; 1(10):590-600

31. Dunnen JT, Dalgleish R, Maglott DR, et al. HGVS Recommendations for the Description of Sequence Variants: 2016 Update. Hum Mutat. 2016;37(6):564-569.

32. Häberle J, Kehrel B, Ritter J, Jürgens $H$ Lämmle B, Furlan M. New strategies in diagnosis and treatment of thrombotic thrombocytopenic purpura: case report and review. Eur J Pediatr. 1999;158(11):883-887.

33. Scully M, Starke R, Lee R, Mackie I, Machin $\mathrm{S}$, Cohen H. Successful management of pregnancy in women with a history of thrombotic thrombocytopaenic purpura. Blood Coagul Fibrinolysis. 2006;17(6):459-463.

34. Furlan M, Robles R, Galbusera M, et al. von Willebrand factor-cleaving protease in thrombotic thrombocytopenic purpura and the hemolytic-uremic syndrome. N Engl J Med. 1998;339(22):1578-1584

35. Saha M, McDaniel JK, Zheng XL. Thrombotic thrombocytopenic purpura: pathogenesis, diagnosis and potential novel therapeutics. Thromb Haemost. 2017; 15(10):1889-1900.

36. Joly BS, Coppo P, Veyradier A. Thrombotic thrombocytopenic purpura. Blood. 2017; 129(21):2836-2846

37. Matsumoto M, Kokame K, Soejima K, et al. Molecular characterization of ADAMTS13 gene mutations in Japanese patients with Upshaw-Schulman syndrome. Blood.
2004;103(4):1305-1310.

38. Kokame K, Nobe Y, Kokubo Y, Okayama A, Miyata T. FRETS-VWF73, a first fluorogenic substrate for ADAMTS13 assay. $\mathrm{Br}$ Haematol. 2005;129(1):93-100

39. Deford CC, Reese JA, Schwartz LH, et al Multiple major morbidities and increased mortality during long-term follow-up after recovery from thrombotic thrombocytopenic purpura. Blood. 2013;122(12):2023-2029.

40. Falter T, Schmitt V, Herold S, et al. Depression and cognitive deficits as long-term consequences of thrombotic thrombocytopenic purpura. Transfusion. 2017; 57(5):1152-1162.

41. Furlan M, Robles R, Solenthaler M, Wassmer M, Sandoz P, Lämmle B. Deficient activity of von Willebrand factor-cleaving protease in chronic relapsing thrombotic thrombocytopenic purpura. Blood. 1997;89(9):30973103.

42. Remuzzi G, Galbusera M, Noris M, et al. von Willebrand factor cleaving protease (ADAMTS13) is deficient in recurrent and familial thrombotic thrombocytopenic purpura and hemolytic uremic syndrome. Blood. 2002;100(3):778-785

43. Fan X, Kremer Hovinga JA, Shirotani-Ikejima $\mathrm{H}$, et al. Genetic variations in complement factors in patients with congenital thrombotic thrombocytopenic purpura with renal insufficiency. Int J Hematol. 2016;103(3):283-291.

44. Bendapudi PK, Hurwitz S, Fry A, et al. Derivation and external validation of the PLASMIC score for rapid assessment of adults with thrombotic microangiopathies: a cohort study. Lancet Haematol. 2017;4(4) e157-e164.

45. Camilleri RS, Cohen H, Mackie IJ, et al Prevalence of the ADAMTS-13 missense mutation R1060W in late onset adult thrombotic thrombocytopenic purpura. J Thromb Haemost. 2008;6(2):331-338.

46. Furlan M, Robles R, Morselli B, Sandoz P, Lämmle B. Recovery and half-life of von Willebrand factor-cleaving protease after plasma therapy in patients with thrombotic thrombocytopenic purpura. Thromb Haemost. 1999;81(1):8-13.

47. Fujimura Y, Kokame K, Yagi $\mathrm{H}$, Isonishi $\mathrm{A}$ Matsumoto M, Miyata T. Hereditary Deficiency of ADAMTS13 Activity: Upshaw-Schulman Syndrome. In: Rodgers GM, ed. ADAMTS13: Biology and Disease. Cham: Springer International Publishing, 2015:73-90

48. Scully M, Knöbl P, Kentouche K, et al Recombinant ADAMTS-13: first-in-human pharmacokinetics and safety in congenital thrombotic thrombocytopenic purpura. Blood. 2017;130(19):2055-2063

49. Rurali E, Banterla F Donadelli $R$, et al. ADAMTS13 Secretion and Residual Activity among Patients with Congenital Thrombotic Thrombocytopenic Purpura with and without Renal Impairment. Clin J Am Soc Nephrol. 2015;10(11):2002-2012.

50. Shang D, Zheng XW, Niiya M, Zheng XL. Apical sorting of ADAMTS13 in vascular endothelial cells and Madin-Darby canine kidney cells depends on the CUB domain and their association with lipid rafts. Blood. 2006;108(7):2207-2215 